En France, le programme Investissements d'Avenir a permis de lancer la construction de "REFIMEVE+ », un équipement d'excellence qui vise à délivrer une référence de fréquence optique à une vingtaine de laboratoires répartis sur tout le territoire métropolitain, via le réseau Internet de RENATER.

Cette nouvelle technologie dépasse de plusieurs ordres de grandeur les capacités offertes par le service GPS dans le domaine radiofréquence (RF). Une révolution est en marche, avec une finalité : ouvrir la voie à des applications inédites dans des domaines aussi variés que la métrologie, la physique ou la géodésie !

\section{La lumière des meilleures horloges accessible par les fibres du réseau Internet}

Anne Amy-Klein ${ }^{(1)}$ (anne.amy-klein@univ-paris13.fr), Olivier Lopez ${ }^{(1)}$, Christian Chardonnet ${ }^{(1)}$, Émilie Camisard ${ }^{(2)}$, Giorgio Santarelli ${ }^{(3)}$ et Paul-Éric Pottie ${ }^{(4)}$

(1) Laboratoire de Physique des Lasers (LPL), Université Paris 13, Sorbonne Paris Cité,

CNRS, 99 avenue Jean-Baptiste Clément, 93430 Villetaneuse

(2) RENATER, 23-25 rue Daviel, 75013 Paris

(3) Laboratoire Photonique, Numérique et Nanosciences, UMR 5298 Université de Bordeaux,

Institut d'Optique Graduate School et CNRS, 1 rue F. Mitterand, 33400 Talence

(4) LNE-SYRTE, Observatoire de Paris, PSL Research University, CNRS, Sorbonne Universités, Université Paris 6 ,

61 avenue de l'Observatoire, 75014 Paris
Jusque récemment, pour comparer quotidiennement la fréquence de deux horloges atomiques installées à grande distance l'une de l'autre, les métrologues faisaient appel à des réseaux de satellites du type GPS. Mais la méthode s'est peu à peu révélée manquer de précision pour comparer les dernières générations d'horloges atomiques, qui fonctionnent avec des lasers pour interroger et manipuler les atomes. On parle alors d'horloges optiques. En effet, la fréquence de ces horloges optiques peut être désormais évaluée avec dix-sept, voire dix-huit chiffres significatifs, tandis que les signaux GPS ne permettent d'en obtenir que quinze ou seize.
Les recherches conduites conjointement au LNE-SYRTE et au LPL ont pour objectif de mettre au point une technique de lien optique destinée à transférer une référence de fréquence par les fibres optiques du réseau Internet, sans dégradation significative de ses performances métrologiques. Entamés il y a dix ans, ces travaux ont permis de démontrer qu'il était possible de transporter un signal d'horloge sur un lien de $86 \mathrm{~km}$, puis d'étendre la longueur de ce dernier progressivement jusqu'à $1500 \mathrm{~km}$. En mesurant le bruit après un aller-retour dans la fibre, nous avons pu nous affranchir presque totalement des perturbations engendrées par ce transport. Les résultats

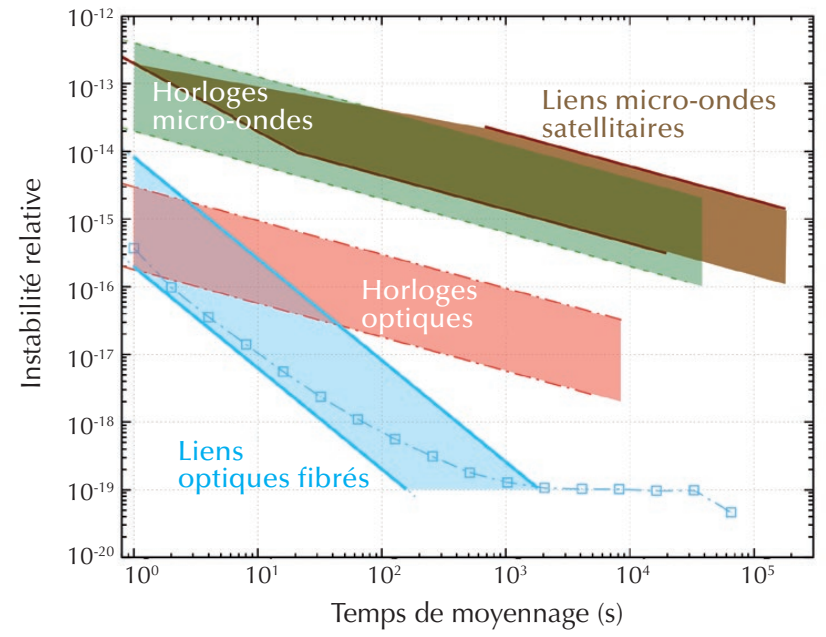

1. Instabilités typiques des différents moyens de comparaison de fréquences (liens optiques de $500 \mathrm{~km}$ ou plus, et liens satellitaires) et des horloges micro-ondes et optiques, en fonction du temps de moyennage (la durée sur laquelle on mesure la fréquence). La courbe bleue en pointillés correspond à l'instabilité mesurée sur un lien optique fibré de 1100 km entre Paris et Nancy et retour. 
>>

sont époustouflants : les fréquences optiques peuvent être transférées avec dix-neuf chiffres significatifs, bien au-delà des performances des meilleures horloges (fig. 1).

Mais cette nouvelle technique concurrente du GPS ne concerne pas que les métrologues. De nombreuses expériences nécessitent une référence de fréquence très stable et dont la valeur soit " exacte ", c'est-à-dire calibrée (ou, plus rigoureusement, "étalonnée ») par rapport à un étalon de fréquence. Par exemple, si l'on souhaite mesurer la fréquence d'absorption d'un atome ou d'une molécule, il nous faut connaître la calibration en fréquence de notre spectromètre. De même, lorsque nous utilisons un générateur de fréquence pour piloter un modulateur par exemple, l'incertitude sur la fréquence RF générée dépend ultimement des performances du résonateur à quartz interne à notre appareil. Aussi, lorsqu'on souhaite faire des mesures de très haute précision, il faut viser une excellente calibration des diverses sources de fréquence et, pour cela, il faut les comparer à un étalon, qui met en pratique la définition de l'unité de fréquence avec une incertitude qui peut être réduite au seizième chiffre significatif.

Mais de tels étalons de fréquence sont coûteux et complexes à mettre en œuvre, d'où l'idée de disséminer leur fréquence à partir du LNE-SYRTE, laboratoire national de métrologie, vers l'ensemble du territoire français.

\section{Comment ça marche}

Les techniques de lien optique consistent à transférer par fibre optique le signal d'un laser ultrastable, dont la fréquence constitue le signal métrologique. Il s'agit d'un laser émettant à la longueur d'onde de $1,55 \mu \mathrm{m}$, à l'intérieur de la bande de fréquence utilisée par les réseaux de télécommunications optiques à longue distance. La fréquence de ce laser est stabilisée sur une cavité Fabry-Perot ultrastable et peut être référencée aux horloges optiques et micro-ondes du SYRTE à l'aide d'un peigne de fréquence. Ainsi, on peut contrôler la fréquence du laser à un niveau meilleur que $10^{-15}$ en valeur relative. Une partie de la lumière du laser ultrastable est injectée dans la fibre optique. Cependant, les fluctuations thermiques et le bruit acoustique perturbent le temps de propagation de la lumière dans la fibre et induisent des fluctuations de la phase et donc de la fréquence transmise. Pour compenser ce bruit, on mesure les fluctuations de la phase du signal transmis après un aller-retour dans la fibre et on les compense avec un système électronique (fig. 2). Cela permet de corriger les bruits réciproques - identiques à l'aller et au retour - et de transmettre le signal ultrastable quasiment sans dégradation.

Cette technique de compensation du bruit de phase a d'abord été développée pour des liens optiques utilisant des fibres dédiées, en France et à l'étranger. Cependant, ces développements étaient freinés par le coût très important de l'accès aux fibres, qui ne permet pas d'envisager la construction de réseaux fibrés métrologiques à l'échelle nationale ou internationale. Nous avons donc proposé d'exploiter l'infrastructure de fibres qui relie déjà tous nos laboratoires, grâce au réseau académique national RENATER. Le principe est de multiplexer en longueur d'onde le signal métrologique avec les signaux optiques qui transportent les données numériques de télécommunications. Ces signaux optiques sont transmis sur des canaux différents, c'est-à-dire à des fréquences proches mais différentes, si bien que leurs propagations seront indépendantes et qu'il n'y aura pas d'interférences. On retrouve ici les principes classiques du multiplexage en longueur d'onde des réseaux de télécommunications optiques. Nous avons donc établi une collaboration avec RENATER et montré qu'il

Laboratoire de métrologie

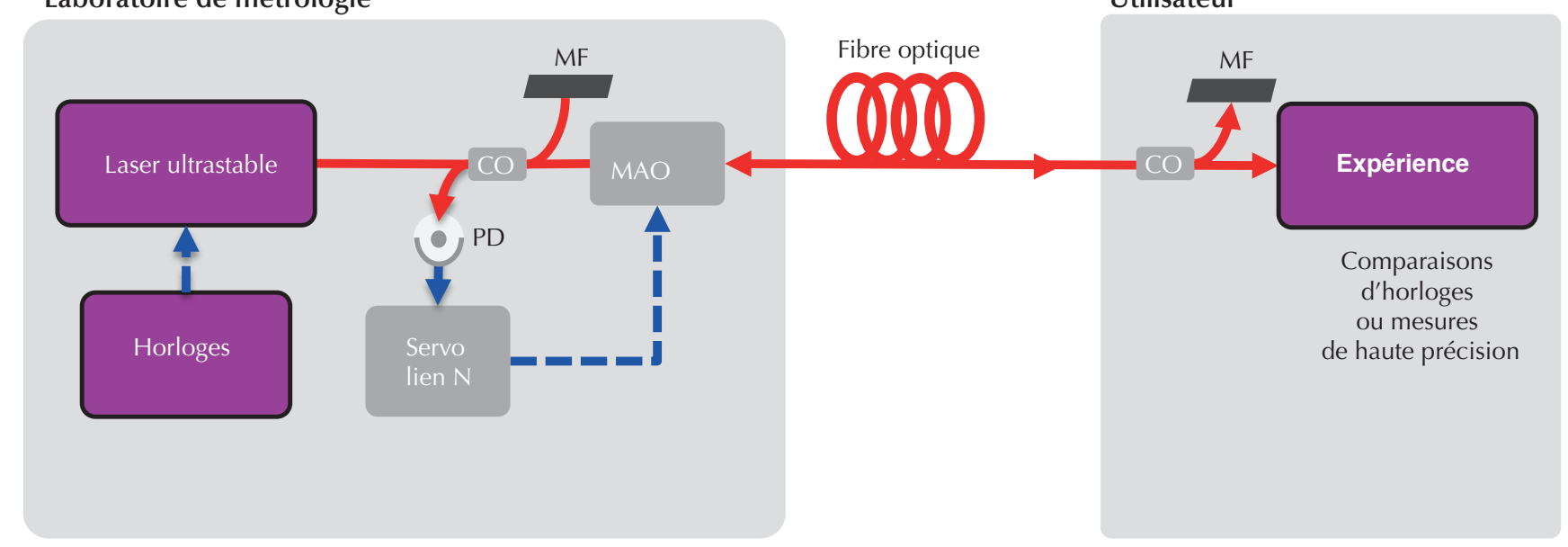

2. Principe d'un lien optique. Le laser ultrastable du laboratoire de métrologie est transmis par fibre optique au laboratoire distant (utilisateur), où une partie du signal est renvoyée afin de détecter les instabilités de phase ajoutées lors de la propagation dans la fibre. Les miroirs de Faraday (MF) permettent de réaliser un interféromètre de Michelson très déséquilibré et de contrôler la polarisation des faisceaux. Un modulateur acousto-optique (MA0) est utilisé pour appliquer la correction du bruit. PD : photodiode, CO : coupleur optique. 
était effectivement possible de transporter dans la même fibre en parallèle le signal métrologique et les données numériques, sans perturbation de ces signaux.

\section{Un réseau national métrologique}

En 2012, nous avons été sélectionnés parmi les projets candidats au programme Investissements d'Avenir, et nous avons entrepris la construction d'un réseau métrologique national, "REFIMEVE+ ", afin de délivrer un signal métrologique élaboré par le LNE-SYRTE à une vingtaine de laboratoires répartis sur tout le territoire métropolitain via le réseau Internet de RENATER (fig. 4, p. 94). Nous sommes en train de transférer notre savoir-faire à un consortium de trois entreprises, qui construiront le matériel spécifique nécessaire pour ce réseau à partir de nos prototypes. Il s'agit essentiellement d'amplificateurs optiques bidirectionnels compatibles avec notre technique de compensation du bruit de propagation, de multiplexeurs pour insérer et extraire le signal métrologique dans le réseau RENATER et de stations de régénération. Ces stations permettent de cascader plusieurs liens optiques, en répétant le signal métrologique d'un lien au suivant (fig. 3). Elles sont installées tous les 200 à $500 \mathrm{~km}$, pour pallier les pertes du réseau et pour réaliser une architecture multipoints permettant une distribution à plusieurs utilisateurs. Tous ces appareils devront bien évidemment pouvoir fonctionner de façon automatique et être pilotables à distance.

Les applications de cette distribution de fréquence sont variées : mesure de constantes fondamentales, tests de violation de parité sur des molécules chirales, études spectroscopiques d'intérêt atmosphérique, tests du principe d'équivalence... En exploitant un lien en forme de boucle, on peut également réaliser un gyromètre géant et explorer sa sensibilité aux phénomènes sismiques par exemple. Enfin, la possibilité d'accéder à une référence de fréquence ultrastable, jusqu'ici réservée à la communauté restreinte des métrologues, suscitera certainement de nouveaux projets dans de nombreux domaines de la physique.

\section{Vers une extension européenne et un réseau international d'horloges}

Nos développements ont permis d'établir plusieurs liens optiques en région parisienne et jusqu'à Strasbourg. Ce dernier lien est constitué de quatre tronçons reliés par des stations de régénération, de Paris à Reims, puis à Strasbourg, puis retour vers Reims et Paris dans une deuxième fibre parallèle à la première, soit une distance totale de $1450 \mathrm{~km}$ environ. Ainsi, les deux extrémités du lien sont situées au LNE-SYRTE (ou au LPL), où nous avons donc pu évaluer les performances du lien. Nous avons ainsi démontré que la fréquence du signal transféré était bien identique à celle du signal injecté, avec une incertitude relative de seulement quelques $10^{-19}$ (c'està-dire au niveau du dix-neuvième chiffre significatif). Nous avons aussi montré que le bruit de phase était bien corrigé dans la limite d'une bande passante de l'ordre de $100 \mathrm{~Hz}$, si bien que la stabilité de fréquence du signal injecté n'était pas dégradée par la propagation dans la fibre. Pour obtenir ces résultats, nous avons dû faire une étude très minutieuse des différentes sources de bruit et des limites fondamentales et technologiques de la réjection de ces bruits. Nous n'avons trouvé aucun déplacement de fréquence jusqu'à présent. L'excellente rejection du bruit que nous avons atteinte nous permet d'évaluer aujourd'hui l'impact des bruits non réciproques, c'est-à-dire qui affectent différemment la phase du signal lors de la propagation aller et retour dans la même fibre (dispersion de mode de polarisation, effet Sagnac, bruit optique de détection...).

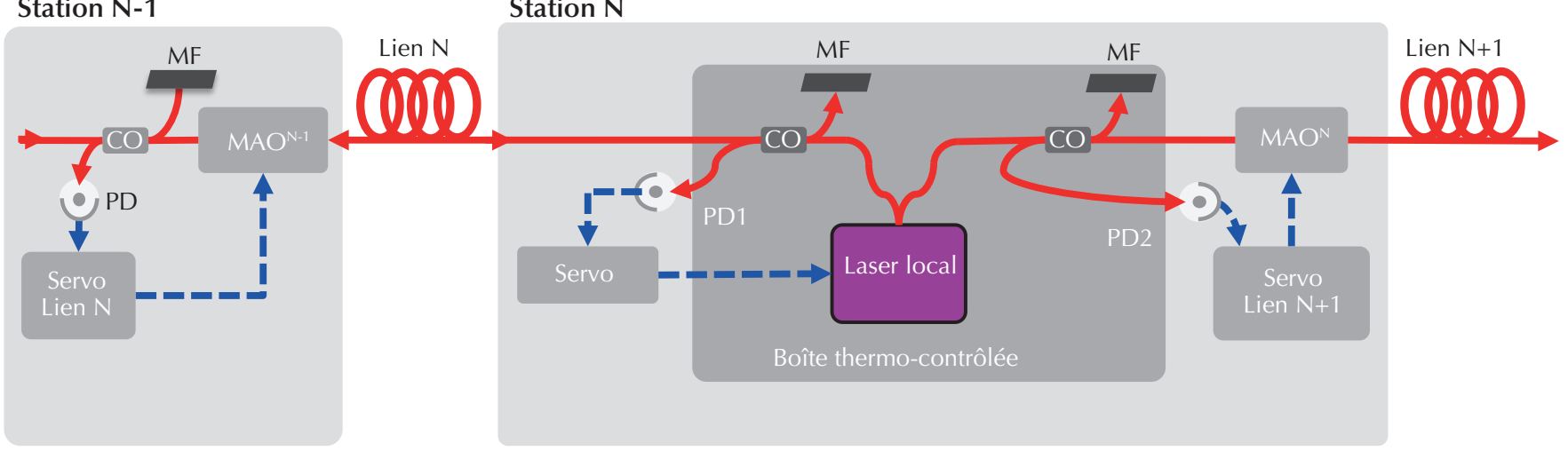

3. Schéma d'une station de régénération du signal métrologique. Le laser local de la station est asservi sur le signal arrivant par le lien amont et est injecté à la fois dans les fibres amont et aval pour permettre les corrections des deux liens amont et aval. Des modulateurs acousto-optiques (MA0) sont utilisés pour appliquer les corrections. Le contrôle de la polarisation des faisceaux est réalisé grâce aux miroirs de Faraday (MF) et à un contrôleur de polarisation (non représenté). PD : photodiode. C0 : coupleur optique. 
\〉

Ces liens optiques se sont également développés dans d'autres pays, essentiellement en Europe et en Asie. Ils utilisent majoritairement des fibres dédiées, sans transfert de données en parallèle, pour relier de grands laboratoires ou instituts de métrologie, la création d'un réseau national étant pour l'instant une spécificité française.

Par exemple, les chercheurs du laboratoire de métrologie allemand, le PTB, situé à Braunschweig, ont démontré dès 2012 un lien optique remarquable de plus de $900 \mathrm{~km}$ en collaboration avec le groupe de T. Hänsch (prix Nobel 2005) à Munich, pour réaliser des mesures de précision sur l'hydrogène atomique.

Plus récemment, nos collègues italiens ont réalisé une mesure très précise de la fréquence d'une horloge à ytterbium atomique entre l'institut de métrologie italien (INRIM) à Turin et le laboratoire européen LENS à Florence, à travers un lien de 642 km (fig. 4).

Dans ce contexte, plusieurs groupes travaillent pour établir un réseau métrologique européen qui relierait les instituts nationaux de métrologie. Ainsi, nous avons établi tout récemment un lien fibré de deux fois $700 \mathrm{~km}$ entre le PTB, à Braunschweig, et le LNE-SYRTE, à Paris (la partie française utilisant le réseau RENATER) et comparé les horloges à atomes froids de strontium des deux laboratoires avec une incertitude inégalée de $510^{-17}$, complètement inaccessible avec le GPS. Une telle mesure, réalisée sur deux horloges développées complètement indépendamment, appuie de façon indiscutable les évaluations des incertitudes des horloges. À moyen terme, cela permettra de mesurer l'effet de gravitation d'Einstein sur la fréquence des horloges, qui est décalée d'environ $10^{-18}$ par $\mathrm{cm}$ d'altitude équivalente, et ainsi de dresser la carte du potentiel gravitationnel terrestre au centimètre près. De telles comparaisons d'horloges distantes ouvrent aussi de nouvelles possibilités pour évaluer les performances, attendues au niveau de quelques $10^{-17}$, du lien micro-onde de l'ensemble d'horloges ACES, qui sera prochainement installé sur la station spatiale internationale pour des tests très contraignants de relativité générale.

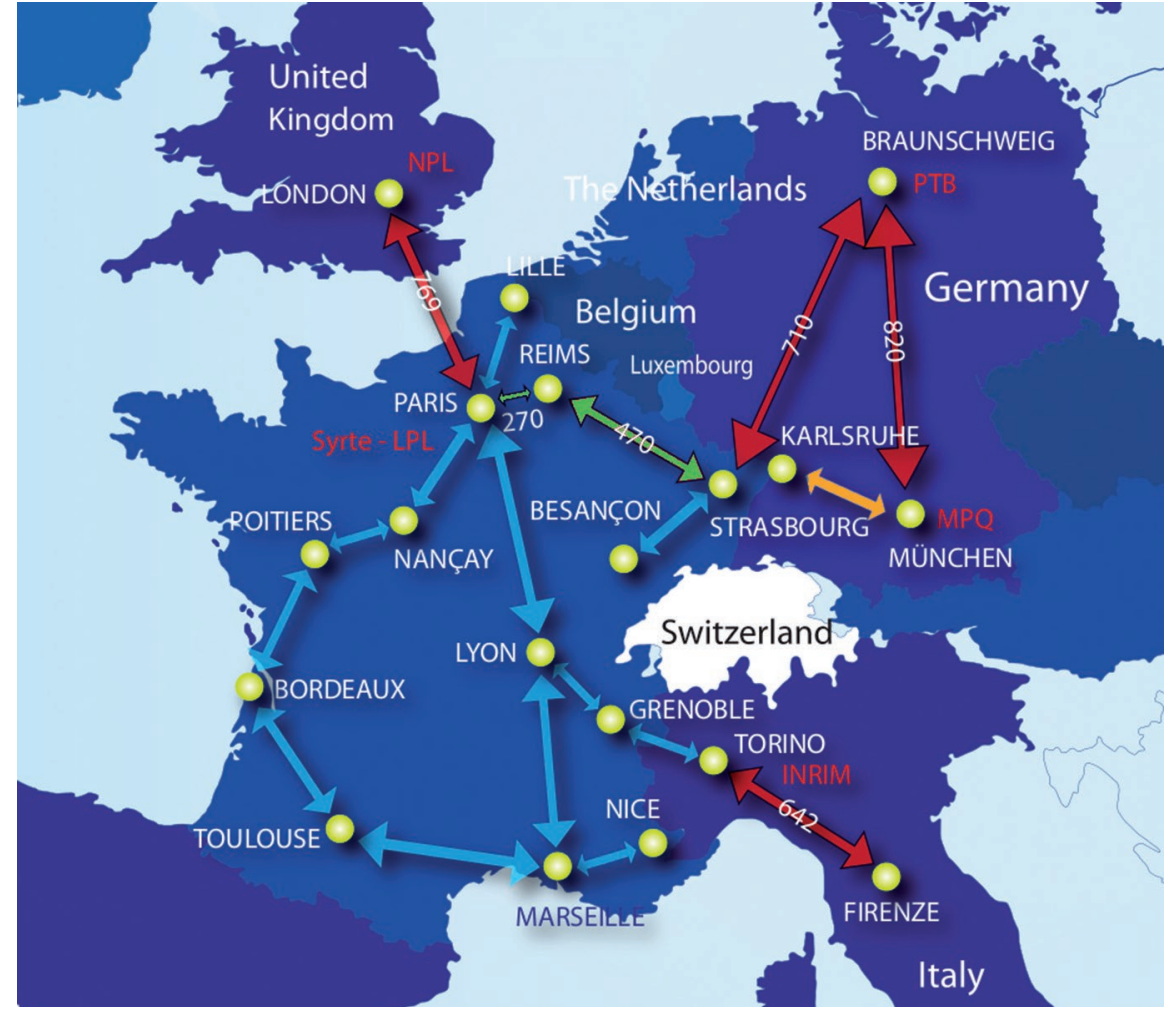

4. Schéma du réseau national REFIMEVE+, en cours de développement, avec des connexions vers les grands centres de métrologie européens. Les liens déjà établis sur le réseau RENATER apparaissent en vert, les autres liens en projet du réseau REFIMEVE+ sont représentés en bleu et dissémineront le signal d'horloge du LNE-SYRTE vers une vingtaine de laboratoires ou établissements français. Les liens qui utilisent des fibres dédiées sont représentés en rouge (liens en fonctionnement) ou en orange (pour les projets). II s’agit de liens à longue distance qui relient les principaux centres européens de recherche en métrologie et en physique de précision. Les points jaunes indiquent les dispositifs optoélectroniques de compensation du bruit des liens et, pour la partie française, de régénération du signal optique.

\section{Conclusion}

Les liens optiques se sont développés très rapidement depuis une dizaine d'années et permettent aujourd'hui de transférer une référence ultrastable de fréquence avec une incertitude qui dépasse de plusieurs ordres de grandeur les capacités du GPS.

En établissant ces liens sur les fibres optiques du réseau RENATER de l'enseignement supérieur et de la recherche, nous avons pour objectif de généraliser l'accès à des signaux de qualité métrologique pour stimuler le développement de très nombreuses expériences de précision en physique. En particulier, les comparaisons des meilleures horloges optiques permettront de mesurer au centimètre près les différences de potentiel gravitationnel terrestre entre deux points distants, ou de tester l'éventuelle variation dans le temps et dans l'espace des constantes fondamentales. Ces comparaisons pourront même permettre d'observer pour la première fois les effets prévus dans certains modèles d'une interaction entre matière "classique " ("hadronique") et matière "noire ".

\section{Références}

\section{Sur les liens optiques}

- Site web du LNE-SYRTE

https://syrte.obspm.fr/spip/science/fop/experiences/ article/liens-optiques-ultra-stables

- Site web du projet REMIFEVE+ :

www.remifeve.fr/

- G. Santarelli et al., Revue Française de Métrologie, 33 (2014) 9-18.

\section{Sur la comparaison d'horloges par peigne de fréquences}

- S. Cundiff et al., Pour la Science, 368 (juin 2008).

- A. Amy-Klein, Reflets de la physique 21 / Le Bup 927 (2010) 42-45.

\section{Sur les horloges optiques}

- https://en.wikipedia.org/wiki/Atomic_clock

-X. Baillard et al., Revue Française de Métrologie,

17 (2009) 11-27.

- F. Riehle, Comptes Rendus Physique 16 (2015) 506-515. 